\title{
Nomenclature for congenital and paediatric cardiac disease: Historical perspectives and The International Pediatric and Congenital Cardiac Code
}

Rodney C.G. Franklin, ${ }^{1}$ Jeffrey Phillip Jacobs, ${ }^{2}$ Otto N. Krogmann, ${ }^{3}$ Marie J. Béland, ${ }^{4}$ Vera D. Aiello, Steven D. Colan, ${ }^{6}$ Martin J. Elliott, ${ }^{7}$ J. William Gaynor, ${ }^{8}$ Hiromi Kurosawa, ${ }^{9}$ Bohdan Maruszewski, ${ }^{10}$ Giovanni Stellin, ${ }^{11}$ Christo I. Tchervenkov, ${ }^{12}$ Henry L. Walters III, ${ }^{13}$ Paul Weinberg, ${ }^{14}$ Robert H. Anderson ${ }^{15}$

${ }^{1}$ Paediatric Cardiology Directorate, Royal Brompton E Harefield NHS Trust, Harefield, Middlesex, United Kingdom; ${ }^{2}$ The Congenital Heart Institute of Florida (CHIF), Division of Thoracic and Cardiovascular Surgery, All Children's Hospital and Children's Hospital of Tampa, University of South Florida College of Medicine, Cardiac Surgical Associates (CSA), Saint Petersburg and Tampa, Florida, United States of America; ${ }^{3}$ Paediatric Cardiology CHD, Heart Center Duisburg, Duisburg, Germany; ${ }^{4}$ Division of Pediatric Cardiology, The Montreal Children's Hospital of the McGill University Health Centre, Montréal, Quebec, Canada; ${ }^{5}$ Heart Institute (InCor), Sao Paulo University School of Medicine, Sao Paulo, Brazil; ' Department of Cardiology, Children's Hospital, Boston, Massachusetts, United States of America; ${ }^{7}$ Cardiac Unit, Great Ormond Street Hospital for Children, London, United Kingdom; ${ }^{8}$ Cardiac Surgery, Children's Hospital of Philadelphia, Philadelphia, Pennsylvania, United States of America; ${ }^{9}$ Cardiovascular Surgery, Heart Institute of Japan, Tokyo Women's Medical University, Tokyo, Japan; ${ }^{10}$ The Children's Memorial Health Institute, Department of Cardiothoracic Surgery, Warsaw, Poland; ${ }^{11}$ Pediatric Cardiac Surgery Unit - University of Padova Medical School, Padova, Italy; ${ }^{12}$ Division of Pediatric Cardiovascular Surgery, The Montreal Children's Hospital of the McGill University Health Centre, Montréal, Quebec, Canada; ${ }^{13}$ Children's Hospital of Michigan, Wayne State University School of Medicine, Detroit, Michigan; ${ }^{14}$ Division of Pediatric Cardiology, The Children's Hospital of Philadelphia, Pennsylvania, United States of America; ${ }^{15}$ Cardiac Unit, Institute of Child Health, Great Ormond Street Hospital for Children, London, United Kingdom

Abstract Clinicians working in the field of congenital and paediatric cardiology have long felt the need for a common diagnostic and therapeutic nomenclature and coding system with which to classify patients of all ages with congenital and acquired cardiac disease. A cohesive and comprehensive system of nomenclature, suitable for setting a global standard for multicentric analysis of outcomes and stratification of risk, has only recently emerged, namely, The International Paediatric and Congenital Cardiac Code. This review, will give an historical perspective on the development of systems of nomenclature in general, and specifically with respect to the diagnosis and treatment of patients with paediatric and congenital cardiac disease. Finally, current and future efforts to merge such systems into the paperless environment of the electronic health or patient record on a global scale are briefly explored.

On October 6, 2000, The International Nomenclature Committee for Pediatric and Congenital Heart Disease was established. In January, 2005, the International Nomenclature Committee was constituted in Canada as The International Society for Nomenclature of Paediatric and Congenital Heart Disease. This International Society now has three working groups. The Nomenclature Working Group developed The International Paediatric and Congenital Cardiac Code and will continue to maintain, expand, update, and preserve this International Code. It will also provide ready access to the International Code for the global paediatric and congenital cardiology and cardiac surgery communities, related disciplines, the healthcare industry, and governmental agencies, both

Correspondence to: Dr Rodney C.G. Franklin, Paediatric Cardiologist, Harefield Site, Royal Brompton and Harefield NHS Trust, Harefield, Middlesex UB9 6JH, UK. Tel/fax: 01895 828659; E-mail: r.franklin@rbht.nhs.uk 
electronically and in published form. The Definitions Working Group will write definitions for the terms in the International Paediatric and Congenital Cardiac Code, building on the previously published definitions from the Nomenclature Working Group. The Archiving Working Group, also known as The Congenital Heart Archiving Research Team, will link images and videos to the International Paediatric and Congenital Cardiac Code. The images and videos will be acquired from cardiac morphologic specimens and imaging modalities such as echocardiography, angiography, computerized axial tomography and magnetic resonance imaging, as well as intraoperative images and videos.

Efforts are ongoing to expand the usage of The International Paediatric and Congenital Cardiac Code to other areas of global healthcare. Collaborative efforts are underway involving the leadership of The International Nomenclature Committee for Pediatric and Congenital Heart Disease and the representatives of the steering group responsible for the creation of the 11th revision of the International Classification of Diseases, administered by the World Health Organisation. Similar collaborative efforts are underway involving the leadership of The International Nomenclature Committee for Pediatric and Congenital Heart Disease and the International Health Terminology Standards Development Organisation, who are the owners of the Systematized Nomenclature of Medicine or "SNOMED".

The International Paediatric and Congenital Cardiac Code was created by specialists in the field to name and classify paediatric and congenital cardiac disease and its treatment. It is a comprehensive code that can be freely downloaded from the internet (http://www.IPCCC.net) and is already in use worldwide, particularly for international comparisons of outcomes. The goal of this effort is to create strategies for stratification of risk and to improve healthcare for the individual patient. The collaboration with the World Heath Organization, the International Health Terminology Standards Development Organisation, and the healthcare industry, will lead to further enhancement of the International Code, and to its more universal use.

Keywords: Databases; cardiac; nomenclature; congenital heart disease

C INICIANS WORKING IN THE FIELD OF CONGENITAL and paediatric cardiology have long felt the need for a common diagnostic and therapeutic nomenclature and coding system with which to classify patients of all ages with congenital and acquired cardiac disease. Over the last 10 years, this desire has been heightened by the need to provide national and international comparisons of surgical results between centres caring for these patients following high profile enquiries such as that examining the outcome of paediatric cardiac surgery at the unit in Bristol, in the United Kingdom, as well as similar events in Sydney Australia, ${ }^{3}$ Winnipeg Canada, ${ }^{4}$ and Denver Colorado, in the United States of America. ${ }^{5-11}$ In order to incorporate effective clinical governance and best practice into our speciality, this requires the harvesting of accurate and validated data on the diagnosis, treatment, and outcome of these patients from prenatal life through to adulthood. Such a system facilitates the comparison of outcomes following interventions between individual units, whilst taking into account the mix of cases involved, accompanying risk factors, and postprocedural complications. Benchmarking against those units who perform best allows analysis of relevant and genuine factors underlying differing outcomes, and instigation of improvements, in terms of both mortality and morbidity. For this to be achieved, it is essential to have a comprehensive and standardised system of coding and classification, using mutually exclusive and unambivalent terms. The system must be easy to use, and also fulfil the needs and expectations of widely different cultures of practice.

Although historically many centres developed their own systems of classification for internal audit, and some co-operative work between centres nationally and across international boundaries has occurred, these systems were dissimilar enough to preclude the large scale studies needed to understand outcomes from the heterogeneous population of patients with congenitally malformed hearts. A cohesive and comprehensive system of nomenclature, suitable for setting a global standard for multicentric analysis of outcomes and stratification of risk, has only recently emerged, namely, The International Paediatric and Congenital Cardiac Code. In this review, we aspire to give an historical perspective on the development of systems of nomenclature in general, and specifically with respect to the diagnosis and treatment of patients with paediatric and congenital cardiac disease. Finally, current and future efforts to merge such systems into the paperless environment of the electronic health or patient record on a global scale are briefly explored. 


\section{History of the classification of disease $\mathrm{P}^{12}$}

Although the gathering of population based information, or censuses, originated in Babylonian times $(3,800 \mathrm{BC})$, it was not until the early 17 th century that the focus shifted away from taxation and military conscription, to causes of death, with the weekly publication from 1603 through the 1830s of the Bills of Mortality in London. ${ }^{13}$ In 1662, these statistics were first systematically analysed by John Graunt, born in 1620, and died in 1674, when he estimated, for instance, the mortality of children before the age of six years to be $36 \%$, an estimate later proven to be quite accurate. In the 18th century François Bossier de Lacroix, born in 1706, and died in 1767, better known as Sauvages, is credited with the first formal classification of diseases based on the methodology of Linnaeus, born in 1707, and died in 1778. This system of classification developed by Sauvages contained 2,400 maladies divided into class, order and species. By the early 19th century, the classification most in use was that of William Cullen, born in 1710, and died in 1790, with four categories:

- "Pyrexiae", in other words pyrexial, or febrile, diseases, such as typhus fever

- "Neuroses", or nervous diseases, such as epilepsy

- "Cachexiae", in other words, cachexial illnesses, or diseases resulting from a bad habit of body, such as scurvy

- "Locales", or local diseases, such as cancer.

In 1837, the General Register Office of England and Wales was established, with William Farr, born in 1807, and died in 1883, as its first medical statistician (Fig. 1). Farr revolutionised the principles of statistical classification and nomenclature, emphasising the need for a common international lexicon to allow the epidemiological study of diseases and thereby their causes, whilst incorporating medical advances. His initial report, published in 1839 , resonates through to the late 20th century:

\footnotetext{
"The advantages of a uniform statistical nomenclature, however imperfect, are so obvious, that it is surprising that no attention been paid to its enforcement in Bills of Mortality. Each disease has, in many instances, been denoted by three or four terms, and each term has been applied to as many different diseases: vague inconvenient names have been employed, or complications registered instead of primary diseases. The nomenclature is of as much importance in this department of inquiry, as weights and measures in the physical sciences, and should be settled without delay."14
}

He later used these methods to help prove the waterborn origin of cholera, the so-called "germ theory", providing evidence that countered the previously

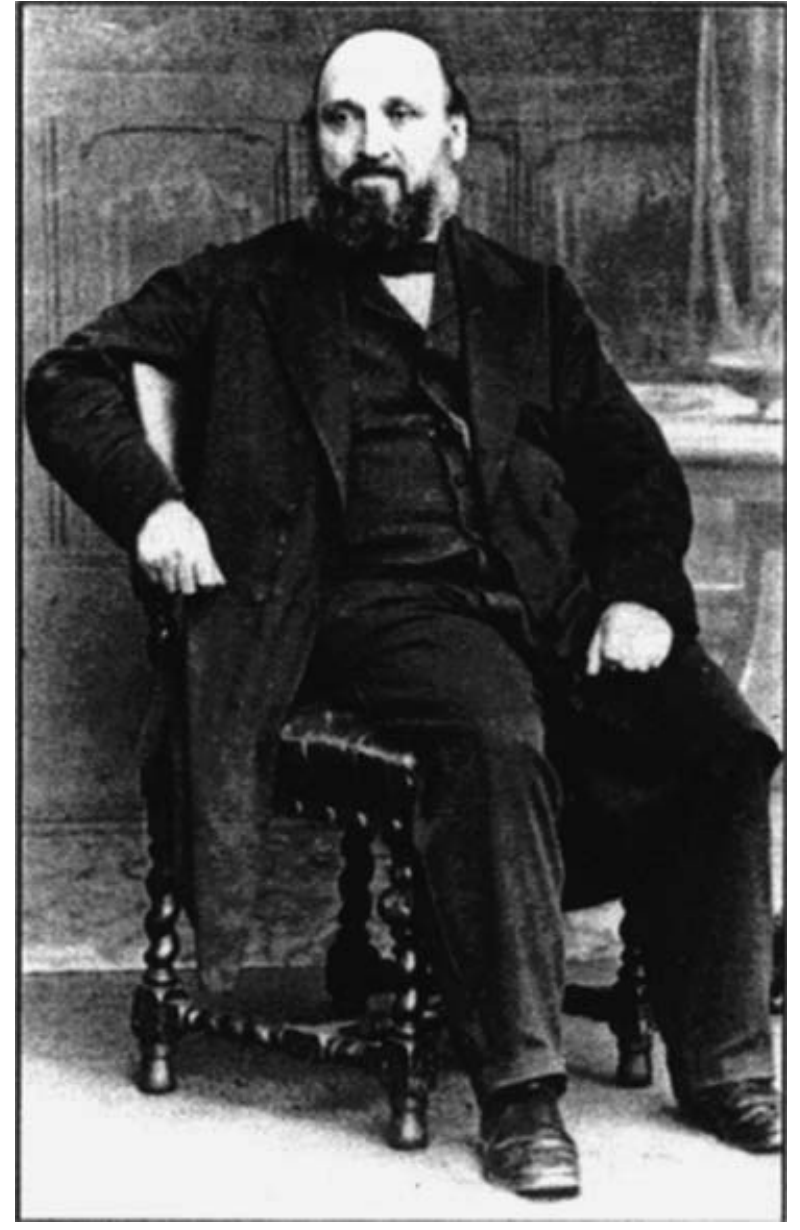

Figure 1.

Dr William Farr, born in 1807, and died in 1883. He was a physician and first medical statistician for the General Register Office of England and Wales from 1839 through 1879. He pioneered the statistical analysis and development of a system of classification for causes of death and disease-related morbidity, emphasising the need for a common international lexicon to allow the epidemiological study of diseases and thereby their causes, whilst incorporating medical advances.

supported miasmatic, or "bad air theory" of disease, leading to the treatment of sewage. The eminence of Farr in the field was recognised at the first International Statistical Congress, held in Brussels in 1853, when he and Marc d'Espine, of Geneva, were asked to prepare an internationally applicable and uniform classification of causes of death. The approach of Marc d'Espine was based on the nature of disease, such as gouty, herpetic, or haematic, whilst the classification proposed by Farr divided into five groups:

- epidemic diseases

- constitutional, or general, diseases

- local diseases arranged according to anatomical site

- developmental diseases

- diseases that are the direct result of violence. 
It was the arrangement suggested by Farr which was dominant in the classification of 139 categories accepted by the Congress in 1855, and over the subsequent 4 revisions through to 1886, particularly the principle of classifying causes of death by anatomical site in distinction to generalised processes of disease. The failure of this classification to achieve wide international recognition, however, led the International Statistical Institute, which had developed from the former Congress, to commission in 1891 a committee chaired by Jacques Bertillon, born in 1851, and died in 1922, Chief of Statistical Services of the City of Paris, to create what became the Bertillon [International] Classification of Causes of Death. This classification was based on the principles propounded by Farr and consisted of three levels of classification, with 44, 99, and 161 titles, respectively. Over the following decades, it was adopted by many countries in the Americas and Europe, with conferences for revision occurring roughly decennially to take note of medical advances.

During the latter half of the 19th century, there was increasing recognition of the need for a parallel list of non-fatal diseases. Farr was again instrumental here, stating the need to:

"extend the same system of nomenclature to diseases which, though not fatal, cause disability in the population, and now figure in the tables of the diseases of armies, navies, hospitals, prisons, lunatic asylums, public institutions of every kind, and sickness societies, as well as in the census of countries like Ireland, where the diseases of all the people are enumerated".

He submitted a list of these entities to the second meeting of the Congress, held in 1856. At the fourth meeting, held in 1860, Florence Nightingale urged its adoption for the tabulation of hospital morbidity in her paper Proposals for a uniform plan of hospital statistics. ${ }^{15}$ In 1900 and 1909, at the first two International Conferences to revise the Bertillon Classification, a parallel classification of diseases for use in statistics of sickness was adopted, but with limited scope. The Health Organisation of the League of Nations, and the International Statistical Institute, recommended at the 4th and 5th conferences for revision, held in 1928 and 1938, respectively, that individual countries develop and promote their own systems of classification for morbidity statistics, using the Causes of Death structure as a template. It was not until the 6th revision conference, held in Paris in 1948, that a single comprehensive list was approved as the International Classification of Diseases, Injuries and Causes of Death. This was endorsed by the First World Health Assembly in the same year, along with rules for selecting a cause of death, and an agreement for international cooperation under the jurisdiction of the recently constituted World Health Organisation.

The 9th Revision of the International Classification of Diseases, in 1975, saw further expansion and structural change, with the addition of a fourth digit, as well as an optional fifth digit to facilitate greater detail where needed by subspecialities. The dagger and asterisk system was introduced to allow the linkage of anatomically specific areas of disease to more generalised diseases. The first International Classification of Procedures in Medicine was also published, in a response to international pressure for a lead in this important area. By the time of the 10th revision, in 1993, the decennial revision schedule was abandoned (Fig. 2) due to the enormous amount of work involved with each revision and an alphanumeric coding structure was introduced. The promised revision of the listing of Procedures in Medicine never materialised, leaving countries to develop further their own systems of classification for interventions. Currently, there are plans in preparation for creating the 11 th revision (see below).

Throughout this time, congenital cardiac diseases have remained very poorly represented. Outdated terminology, inconsistent logic, and little appreciation of the inherent complexity of lesions, has meant that this system of classification has never been robust enough for the purposes of clinical governance. In the 9th and 10th revisions, there are a total of only 29 and 73 individual codes, respectively, for congenital cardiovascular lesions. Despite these limitations, both versions remain in use, mostly for 'billing', returns to central governments, and crude epidemiological surveillance. A comprehensive, clinically acceptable system for the naming and coding of congenital cardiac disease, relevant acquired cardiac disease, and, most importantly, procedures to treat congenital and relevant acquired cardiac lesions, was needed.

\section{Paediatric and congenital cardiac nomenclature and The International Paediatric and Congenital Cardiac Code}

Although there were many descriptive publications of individuals with various congenital cardiac malformations in the 19th and early 20th centuries, it was the publication in 1936 of the Atlas of Congenital Cardiac Disease by the distinguished Canadian physician Maude Abbott, that saw the first systematic classification of congenital cardiac lesions. ${ }^{16}$ Over the following decades, several centres developed more inclusive systems of classification for both the diagnosis and treatment of congenital cardiac disease. It was not until the 1980s that advances in the hardware and software underpinning information technology made 


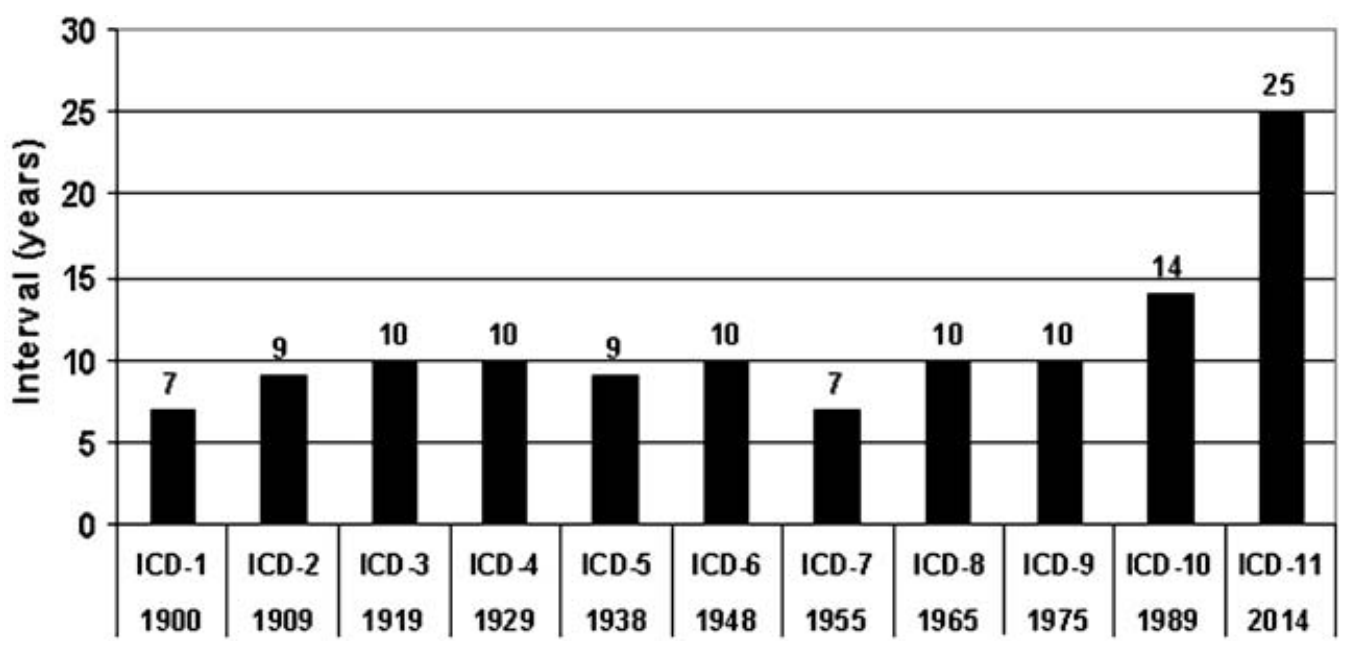

Figure 2 .

This graph documents the time interval between each Revision of the International Classification of Diseases. The Bertillon [International] Classification of Causes of Death was presented by Bertillon at the meeting of the International Statistical Institute in Chicago in 1893 and adopted by the International Statistical Institute. The Bertillon Classification of Causes of Death was adopted by several cities and countries. In 1898, the American Public Health Association recommended the adoption of the Bertillon Classification by registrars of Canada, Mexico, and the United States of America. The Association further suggested that the classification should be revised every ten years. The First International Conference to revise the Bertillon Classification of Causes of Death was held in Paris in 1900. Subsequent revisions occurred as documented in this graph. (This graph is adopted from a presentation made by Dr. T. Bedirhan Üstün at The Ninth Meeting of The International Working Group for Mapping and Coding of Nomenclatures for Paediatric and Congenital Heart Disease [Nomenclature Working Group], Keio Plaza Hotel, Tokyo, JAPAN, Saturday July 7, 2007 - Thursday July 12, 2007.)

it feasible to have a uniform and internationally acceptable system of nomenclature, with facilitation of entry of data and exchange of information.

In Europe, this era was marked by the publication in 1985 of the Brompton Hospital Diagnostic Code, with 507 items classified using an associated six digit code. ${ }^{17}$ This diagnostic system was expanded to 1,717 terms in the Netherlands in the late 1980s, including sections on acquired cardiac disease, arrhythmias, relevant non-cardiac anomalies and, most importantly, surgical and transcatheter procedures. From 1989 through 1994, further enhancements occurred in all areas, with the introduction of terms for postprocedural complications and qualifiers, leading to a single hierarchical tree with over 4,300 terms, each with its own six digit code. ${ }^{18}$ These terms were incorporated into the United Kingdom panmedical nomenclature and coding initiative within the National Health Service, becoming Clinical Terms version 3.1, or the Read Codes, which later formed a fundamental part of the Systematized Nomenclature of Medicine Clinical Terms, also known as "SNOMED-CT", described below. This version of 1994 was used by The European Congenital Heart Surgeons Foundation to audit surgical outcomes across much of Europe. Finally, in 1998 , this system of nomenclature was adopted by The Association for European Paediatric Cardiology as the standard system for databases across Europe, and was titled the European Paediatric Cardiac Code. ${ }^{19}$
The publication of this system included rule-based crossmapping to the 9th and 10th revisions of the International Classification of Diseases. ${ }^{18,19}$

In the late 1990s, a second major and parallel initiative emerged to list and classify paediatric and congenital cardiac diagnoses and procedures. In the mid 1990s, two large multi-institutional surgical database projects were in process:

- The Congenital Heart Surgery Database of The Society of Thoracic Surgeons in North America, and

- The European Congenital Heart Defects Database of the European Congenital Heart Surgeons Foundation, which in 2003 was renamed the European Congenital Heart Surgeons Association. In the mid 1990s, this database received data from 18 countries. In September 1999, during the Annual Meeting of The European Association for Cardio-Thoracic Surgery in Glasgow Scotland, the final decision was made that The European Congenital Heart Defects Database of the European Congenital Heart Surgeons Foundation would also become a part of The European Association for Cardio-Thoracic Surgery Database Project.

Both project teams identified a need for an international structure that would standardize nomenclature and strategies for reporting. There was, for example, disharmony amongst the many centres and 
countries on the terms used to describe identical congenital cardiac lesions, such as subtypes of ventricular septal defects. This led The Society of Thoracic Surgeons, The European Congenital Heart Surgeons Association, and The European Association for CardioThoracic Surgery to set up the International Congenital Heart Surgery Nomenclature and Database Project in $1998 .^{20}$ Over the next two years, a series of meetings between a core group of experts in congenital cardiac surgery and paediatric cardiology met to create a standardized inclusive hierarchical nomenclature, with a generous use of synonyms, based on consensus, scientific principals and popular usage. ${ }^{21}$ This system of nomenclature of the International Congenital Heart Surgery Nomenclature and Database Project contains 5 lists:

- Diagnoses

- Procedures

- Noncardiac Abnormalities

- Preoperative Risk Factors

- Intraoperative and Postoperative Complications.

In early 2000, both of these two systems of nomenclature were published: ${ }^{19,21}$

- The nomenclature of the International Congenital Heart Surgery Nomenclature and Database Project of The Society of Thoracic Surgeons, The European Congenital Heart Surgeons Association, and The European Association for Cardio-Thoracic Surgery

- The European Paediatric Cardiac Code of The Association for European Paediatric Cardiology.

Both systems of nomenclature included a comprehensive Long List, with thousands of terms, and a Short List designed to be used as part of a Minimum Dataset for audit and research purposes, with up to 650 terms. $^{19,21}$ The comprehensive dataset includes all the imagined variables, in a hierarchical structure, and are detailed enough to enable the analyses of stratification of risk. The minimum dataset includes sufficient data points to enable easy and mandatory sharing of interinstitutional data for basic analysis of mortality and interpretation of trends. Both Long Lists map fully to their respective Short Lists. This nearly simultaneous publication of these two complementary systems of nomenclature led to the problematic situation of having two lexicons that were to be widely adopted, with the potential risks of invalidating multicentric projects due to confusion between the two systems and duplicate or inaccurate entries within institutions. A meeting was convened, therefore, between representatives from The Association for European Paediatric Cardiology, The Society of Thoracic Surgeons, and The European Association for Cardio-Thoracic Surgery, which was held on October 6, 2000, in Frankfurt, Germany. It was agreed to establish The International Nomenclature Committee for Pediatric and Congenital Heart Disease, including representatives of the three societies, as well as representatives from Asia and South America, to work in partnership and produce a reconciliatory bidirectional map between the two systems. ${ }^{22}$ Fortuitously, the International Congenital Heart Surgery Nomenclature and Database Project did not feature a numerical code, and it was therefore resolved to use the six digit numerical code derived from the European Paediatric Cardiac Code as the backbone for mapping the two systems. The feasibility of this project was established by the creation of a rule-based bidirectional crossmap between the two Short Lists. ${ }^{23}$ This work was then presented and endorsed at the Third World Congress of Pediatric Cardiology and Cardiac Surgery in Toronto, Canada on May 27, 2001, during the First International Summit on Nomenclature for Congenital Heart Disease, which was attended by representatives from at least ten Societies and five continents. ${ }^{22}$ This bidirectional crossmap between the two Short Lists therefore established the precedent for achieving the main goal of mapping the two comprehensive Long Lists to each other to create the International Paediatric and Congenital Cardiac Code, for subsequent presentation at the Fourth World Congress of Pediatric Cardiology and Cardiac Surgery in Buenos Aires, Argentina, on Monday September 19, 2005.

The working component of this International Nomenclature Committee has been the International Working Group for Mapping and Coding of Nomenclatures for Paediatric and Congenital Heart Disease, with 12 members, better known as the Nomenclature Working Group. $^{24}$ On September 19, 2005, the Nomenclature Working Group was able to report to the Second International Summit on Nomenclature for Congenital Heart Disease at the Fourth World Congress of Pediatric Cardiology and Cardiac Surgery in Buenos Aires, Argentina, that they had met seven times, over a total period of 33 days, and had succeeded in crossmapping the majority of congenital cardiac lesions. ${ }^{25}$ The International Paediatric and Congenital Cardiac Code at this point consisted of 7,623 items, each with a six digit code, in two dominant versions:

- The International Paediatric and Congenital Cardiac Code derived from the European Paediatric Cardiac Code of The Association for European Paediatric Cardiology;

- The International Paediatric and Congenital Cardiac Code derived from the International Congenital Heart Surgery Nomenclature and Database Project of The Society of Thoracic Surgeons, The European Congenital Heart 
Surgeons Association, and The European Association for Cardio-Thoracic Surgery.

In this article, we will refer to The International Paediatric and Congenital Cardiac Code as the International Code. Each unique six digit code in the International Code corresponds to a single entity, whether it be a morphological phenotype, procedure, symptom or genetic syndrome, with the mapped terms in the two versions being synonymous to each other. Additional systems of nomenclature, for paediatric cardiology and cardiac surgery, which are mapped to the common spine, include the Bostonbased Fyler codes, and the Canadian nomenclature system. There is also mapping to the 9th and 10th revisions of the International Classification of Diseases provided by the World Health Organisation, usually in a many to one fashion, given the limitations of the International Classification of Diseases. Two subsequent meetings of the Nomenclature Working Group, in 2006 and 2007, expanded the International Code further when covering the areas of fetal cardiology, arrhythmias, congenital coronary arterial anomalies, echocardiography, and interventional cardiology procedures, with the help of several invited experts. The International Paediatric and Congenital Cardiac Code is available for download without cost from the internet at http://www.IPCCC.net.

The Nomenclature Working Group has also published review articles which provide a unified and comprehensive classification, with definitions, for several complex congenital cardiac malformations, along with a complete listing of the relevant codes and terms in both versions of the International Code:

- the functionally univentricular heart ${ }^{26}$

- hypoplastic left heart syndrome $e^{27}$

- discordant atrioventricular connections ${ }^{28}$ and

- cardiac structures in the setting of heterotaxy. ${ }^{29}$

A separate parallel process has recently also involved members of the Nomenclature Working Group, namely the development of a nomenclature and classification system for complications during and following interventions for patients with paediatric and congenital cardiac disease, along with the creation of consensus definitions for these complications, as supported by The MultiSocietal Database Committee for Pediatric and Congenital Heart Disease. This listing of complications and their definitions is a primary purpose of this Supplement in Cardiology in the Young. Prior to this process, the International Paediatric and Congenital Cardiac Code had a total of 1,422 complication codes listed. This listing of complications in the International Code has now been expanded to over 2,500 items, each with its own numerical six digit code and definition.
Future developments and the International Paediatric and Congenital Cardiac Code

The crossmapping of the Short Lists of the International Congenital Heart Surgery Nomenclature and Database Project and the European Paediatric Cardiac Code, ${ }^{23}$ which are used primarily for analyses of multi-institutional and international outcomes following operations and procedures for patients with congenitally malformed hearts, and which are derived directly from their respective Long Lists, potentially enables the huge number of over 100,000 patients registered in their respective databases to be used together. As discussed elsewhere in this Supplement, there is already work underway to analyse the potential usefulness of stratification of complexity and adjustment for casemix, using the Aristotle Complexity Score and Risk Adjustment in Congenital Heart Surgery system. ${ }^{30}$ The structure and content of the Short Lists remain the purview of the Societies and organizations who created them. It is, of course, possible to shorten further, or create Short Lists specific to a subspecialty, or minimum datasets to suit individual projects and research aims, provided that those using the Long List as the data entry mechanism, focus on similar areas and ensure there are no orphan terms produced during the process of electronic conversion of the terms in the Long List to specific terms in the Short List based on the crossmap.

In January, 2005, the International Nomenclature Committee was constituted in Canada as The International Society for Nomenclature of Paediatric and Congenital Heart Disease. ${ }^{25}$ On July 9, 2007, during its third annual meeting held in Tokyo, Japan, the International Society created two new Working Groups, so that the Society now has the following three committees:

- The International Working Group for Mapping and Coding of Nomenclatures for Paediatric and Congenital Heart Disease, also known as the Nomenclature Working Group. This Group will continue to maintain, develop, expand, update, and preserve The International Paediatric and Congenital Cardiac Code. It will also provide ready access to the International Code for the global paediatric and congenital cardiology and cardiac surgery communities, related disciplines, the healthcare industry, and governmental agencies, both electronically and in published form.

- The International Working Group for Defining the Nomenclatures for Paediatric and Congenital Heart Disease, also known as the Definitions Working Group. This Group will write definitions for the terms in the International Paediatric and Congenital Cardiac Code, building on the 
previously published definitions from the Nomenclature Working Group. ${ }^{25-29}$

- The International Working Group for Archiving and Cataloguing the Images and Videos of the Nomenclatures for Paediatric and Congenital Heart Disease, also known as both the Archiving Working Group and the Congenital Heart Archiving Research Team. This Group will link images and videos to the International Paediatric and Congenital Cardiac Code. The images and videos will be acquired from cardiac morphologic specimens and imaging modalities such as echocardiography, angiography, computerized axial tomography and magnetic resonance imaging, as well as intraoperative images and videos. This archive will be linked to the Cardiothoracic Surgery Network, or "CTSNet", Congenital Portal (http://www.ctsnet.org).

Currently, the use of the International Paediatric and Congenital Cardiac Code is largely limited to individuals working in the field of paediatric cardiology and paediatric cardiac surgery. There is a need to expand this usage to other areas of global healthcare, where the individual with a congenitally malformed heart may be referred for care, or have an impact, such as other medical subspecialties, hospital administrative and insurance systems, and the World Health Organization. On July 8, 2007, in Tokyo, Japan, the International Society for Nomenclature of Paediatric and Congenital Heart Disease met with representatives of the steering group responsible for the creation of the 11th revision of the International Classification of Diseases, administered by the World Health Organisation, and scheduled for launch in 2014. It was agreed in principle that there should be constructive dialogue between the Topic Advisory Group for Rare Diseases, which will cover congenital and paediatric cardiac disease, and the International Society for Nomenclature of Paediatric and Congenital Heart Disease, so that future listings of congenital and relevant acquired cardiac disease can better be represented within the International Classification of Diseases. One option could be to create a specific Short List, or minimum dataset, which could be integrated into the 11th revision, and to which the International Paediatric and Congenital Cardiac Code is mapped. Part of the plans for the 11th revision appears to include incorporation into the electronic health record.

\section{The Electronic Health Record and the Systematized Nomenclature of Medicine or "SNOMED"}

With recent continued advancements in computers and information technology, ${ }^{30}$ in many developed countries the electronic patient record is beginning to become a reality. Healthcare providers now demand computerisation of the healthcare record and clinical support systems, and movement towards a paperless environment. This advance involves creating a standardized system for obtaining accurate and detailed clinical information on the history of the patient, as well as diagnosis, and treatment, using a reliable and easily validated methodology. The hope is that the savings made from this patient-centric system, free from duplication of data entry, would free up resources for comparative studies across units and nations of clinical and cost-related outcomes. This achievement would allow risk-adjusted benchmarking, and identification of best practice, as well as the generation of effective guidelines and tools to support decision-making. The prerequisite for this accomplishment is an underlying, all encompassing, common nomenclature and coding system for healthcare, with clinician-led and validated entry of data, the qualities of which should include the following specifications:

- an "atom" based, clinically sensitive structure, so that each numerical code corresponds to the lowest denominator concept, based on a multidisciplinary clinical knowledgebase. This atomically oriented system would not preclude higher level more complex concepts which are in common clinical use, such as "hypoplastic left heart syndrome"

- the ability to code relevant qualities of severity and complexity, for stratification of risk

- standardisation of underlying terminologies and hierarchical classifications across multiple specialities

- an aim for one preferred representation of a concept or phenotype, but with explicit integration of synonyms and commonly used abbreviations. The user would then have a choice of synonymous terms, enabling entry of data that easily encompasses different cultures of practice and different medical specialities. This use of synonyms should abrogate multiple, same-meaning, redundant codes

- the ability to access terms through multiple intuitive hierarchies, such as concepts for "ventricular septal defect" via pathways based on septal defect, tetralogy of Fallot, or functionally univentricular heart

- routine clinically sensitive updates for new procedures and scientific advances. This specification is the "responsiveness" of the system of nomenclature

- the ability to move a concept to a new hierarchy, whilst maintaining its unique code, in response to scientific or clinical advances, such as genetics or transcatheter procedures 
- the ability to account for diagnostic uncertainty or negative findings, using an additional attribute, such as "suspected", "uncertain" or "ruled out"

- a definition for each term provided by an overseeing expert authority. In this case, we would suggest that the Definitions Working Group of The International Society for Nomenclature of Paediatric and Congenital Heart Disease would oversee definitions for codes related to congenital cardiac disease and acquired paediatric cardiac disease

- the ability to deal with obsolete or scientifically inaccurate concepts by reassignment to the correct term and using an "obsolete" label for the term itself, whilst retaining historical data

- multilingual translations.

These specifications are not available in current international systems, such as the 10th revision of the International Classification of Diseases. As a consequence, many non-integrated self-made, or industry-created, solutions exist, which are often expensive and of variable quality and integrity.

In 1974, the College of the American Pathologists created the Systematized Nomenclature of Medicine from their more restrictive listing of 1965, the Systematised Nomenclature of Pathology, generating an electronic format in 1977. After considerable expansion over the next 25 years, with the endorsement of the National Library of Health and American National Standards Institute, it combined with the Clinical Terms version 3 project based in the United Kingdom to become, in 2002, the Systematized Nomenclature of Medicine Clinical Terms, or "SNOMED-CT". ${ }^{11}$ During this time, and subsequently, it has achieved wide acceptance as an effective tool to classify diseases, and is being promoted as the optimal product for the electronic health record, having most of the above specifications. In a précis of their own words: it is a dynamic, scientifically validated clinical reference terminology that makes health care knowledge more usable and accessible by providing a common language that enables a consistent way of capturing, sharing, and aggregating health data across all specialties and sites of care. There are over 365,000 concepts, 984,000 attributes or descriptors, and 1.5 million connections. International interest exists in its use from over 30 countries worldwide. More recently, in 2007, nine countries have grouped together to purchase the product as the not-for-profit International Health Terminology Standards Development Organisation, whilst having the College of American

\footnotetext{
Australia, Canada, Denmark, Lithuania, New Zealand, Netherlands, Sweden, United Kingdom, United States.
}

Pathologists continuing in a managerial role. The aim is to have a global, validated, and stable system of nomenclature with shared ownership, transparent management processes, and a secure governance structure, as well as financial sustainability. The licensing arrangement with industry vendors is to be simple, clear and inclusive, whilst the user licence is free to member states.

With respect to congenital and paediatric cardiology and surgery, over 4,000 terms are already integrated in to the Systematized Nomenclature of Medicine Clinical Terms due to the amalgamation with the United Kingdom Clinical terms system in 2002, which itself had taken these terms into its structure in 1994, as described above. These 4,000 terms are also a core part of The International Paediatric and Congenital Cardiac Code, with often exact or near-exact matching to the version derived from the European Paediatric Cardiac Code. Unfortunately, many categories also exist with redundant and obsolete terms, as well as areas of non-intuitive hierarchy. This section of the Clinical Terms lexicon has not had input from experts in this field. One of the remits of the International Health Terminology Standards Development Organisation is to promote the development of subsets for individual medical disciplines who already have a system of nomenclature in active use. Initial discussions between the representatives of the International Health Terminology Standards Development Organisation and The International Society for Nomenclature of Paediatric and Congenital Heart Disease have been productive, and there has been agreement that the Organisation will work with the International Society to incorporate fully the International Paediatric and Congenital Cardiac Code into the Systematized Nomenclature of Medicine Clinical Terms, whilst "cleaning up" the latter to a clinically sound product, and mapping to the six digit numerical code. This major work has just commenced at the time of writing.

Discussions are also ongoing with the cardiovascular specialist healthcare industry about its incorporation into echocardiographic and catheterisation based software, either independently or as a specialist subset of the Systematized Nomenclature of Medicine Clinical Terms.

\section{Conclusions}

The International Paediatric and Congenital Cardiac Code was created by specialists in the field to name and classify paediatric and congenital cardiac disease and its treatment. It is a comprehensive code that can be freely downloaded from the internet (http:// www.IPCCC.net) and is already in use worldwide, 
particularly in its Short List formats for international comparisons of outcomes. This latter work is being used to compare performance between units, and even individual clinicians, to create strategies for stratification of risk, and to improve healthcare for the individual patient. Such comparisons have already been shown to be culturally reassuring when no statistically different outcomes can be demonstrated across a nation. ${ }^{32}$ We anticipate that future cooperative multi-institutional studies will enable the optimisation of the quality and effectiveness of healthcare for our patients with congenital cardiac malformations, whilst influencing the allocation of increasingly limited resources.

The collaboration with the World Heath Organization, the International Health Terminology Standards Development Organisation, and the healthcare industry, will lead to further enhancement of the International Code, and to its more universal use. Future work of the International Society for Nomenclature of Paediatric and Congenital Heart Disease, and its three working groups, should produce in the next few years a unique, multifaceted lexicon of terms related to congenital cardiac disease and acquired paediatric cardiac disease, for clinical, governance, educational, research, and administrative purposes. This system will be replete with comprehensive definitions, and the ability to visualise lesions, along with their modes of therapy, across a full array of imaging platforms. All will be available at the click of a mouse, and free throughout the world.

\section{Acknowledgement}

We thank The Children's Heart Foundation (http:// www.childrensheartfoundation.org/) for financial support of this research.

\section{References}

1. Smith R. All changed, changed utterly. Brit Med J 1998; 316: 1917-1918.

2. Learning from Bristol: the report of the public inquiry into children's heart surgery at the Bristol Royal Infirmary 1984-1995. [http:// www.bristol-inquiry.org.uk], accessed October 22, 2004.

3. Wright J. Putting a surgeon under: a personal story of hospital politics. [http://www.uow.edu.au/arts/sts/bmartin/dissent/documents/ Wright], accessed December 24, 2007.

4. The Report of the Manitoba Pediatric Cardiac Surgery Inquest: An Inquest into twelve deaths at the Winnipeg Health Sciences Centre in 1994. [http://www.pediatriccardiacinquest.mb.ca/], accessed October 22, 2004.

5. Sherry A. Children's Hospital cardiology chief told to resign. Denver Post. Article Published: March 1, 2001. [http://www. denverpost.com/news/news0301b.htm], accessed March 21, 2001.

6. Sherry A. Hospitals shield mortality rates. Denver Post. Article Published: March 2, 2001. [http://www.denverpost.com/news/ news0302d.htm], accessed March 21, 2001.
7. The Denver Post Editorial Board. At the heart of the problem. Denver Post. Article Published: March 2, 2001. [http://www. denverpost.com/opinion/edits0302c.htm], accessed March 21, 2001.

8. Johnson L. Baby's death at Children's turns parents to their faith. Denver Post. Article Published: March 3, 2001. [http:// www.denverpost.com/opinion/lett0311.htm], accessed March 21, 2001.

9. White S. Kids' best interests: Re: "Children's Hospital cardiology chief told to resign," March 1. Denver Post. Article Published: March 3, 2001. [http://www.denverpost.com/opinion/lett0311. htm], accessed March 21, 2001.

10. Hernandez J. Other options. Denver Post. Article Published: March 3, 2001. [http://www.denverpost.com/opinion/lett0311. htm], accessed March 21, 2001.

11. Weinberg S. Rare look inside a surgeon's sanctum. Denver Post. Article Published: Sunday, April 20, 2003. [http://www.denverpost. $\mathrm{com} /$ Stories/0\%2C1413\%2C36 28 1333663\%2C00.html], accessed October 22, 2004.

12. Much of this section was derived from: World Heath Organization. History of the development of the ICD. [http:// www.who.int/classifications/icd/en/HistoryOfICD.pdf], accessed December 24, 2007.

13. Greenwood M. Medical statistics from Graunt to Farr. Cambridge University Press, Cambridge, 1948.

14. Farr W. First annual report. Registrar General of England and Wales, London, 1839, p 99.

15. Nightingale F. Proposals for a uniform plan of hospital statistics. Fourth International Statistical Congress, London, 1860.

16. Abbott ME. Atlas of Congenital Cardiac Disease. The American Heart Association, New York, 1936.

17. Miller GAH, Anderson RH, Rigby ML. The diagnosis of congenital heart disease; incorporating the Brompton Hospital Diagnostic Code. Castle House, Tunbridge Wells, 1985, pp 110-120.

18. Franklin RCG, Anderson RH, Daniëls O, et al. The European Paediatric Cardiac Code. Card Young 1999; 9: 633-657.

19. Association for European Paediatric Cardiology. The European Paediatric Cardiac Code. Cardiol Young 2000; 10 (Suppl 1): $1-146$.

20. Mavroudis C, Jacobs JP. Congenital heart surgery nomenclature and database project: overview and minimum dataset. Ann Thorac Surg 2000; 69: S2-S17.

21. Mavroudis C, Jacobs JP (editors). Congenital Heart Surgery Nomenclature and Database Project. Ann Thorac Surg 2000; 69 (Suppl): S1-S372.

22. Béland M, Jacobs JP, Tchervenkov CI, Franklin RCG. The International Nomenclature Project for Paediatric and Congenital Heart Disease: Report from the Executive of The International Working Group for Mapping and Coding of Nomenclatures for Paediatric and Congenital Heart Disease. Cardiol Young 2002; 12: $425-430$.

23. Franklin RCG, Jacobs JP, Tchervenkov CI, Béland MJ. The International Nomenclature Project for Congenital Heart Disease: bidirectional crossmap of the Short lists of the European Paediatric Cardiac Code and the International Congenital Heart Surgery Nomenclature database Project. Card Young 2002; 12: 431-435.

24. Béland MJ, Franklin RCG, Jacobs JP, et al. Update from The International Working Group for Mapping and Coding of Nomenclatures for Paediatric and Congenital Heart Disease. Cardiol Young 2004; 14: 225-229.

25. Franklin RC, Beland MJ, Krogmann ON. Mapping and coding of nomenclatures for paediatric and congenital heart disease. Cardiol Young 2006; 16: 105-106.

26. Jacobs JP, Franklin RCG, Jacobs ML, et al. Classification of the functionally univentricular heart: unity from mapped codes. Cardiol Young 2006; 16 (Suppl 1): 9-21. 
27. Tchervenkov CI, Jacobs JP, Weinberg PM, et al. The nomenclature, definition and classification of hypoplastic left heart syndrome. Cardiol Young 2006; 16: 339-368.

28. Jacobs JP, Franklin RC, Wilkinson JL, et al. The nomenclature, definition and classification of discordant atrioventricular connections. Cardiol Young 2006; 16 (Suppl 3): 72-84; Review.

29. Jacobs JP, Anderson RH, Weinberg P, et al. The nomenclature, definition and classification of cardiac structures in the setting of heterotaxy. Cardiol Young 2007; 17 (Suppl 2): 1-28.
30. Jacobs JP, Maruszewski B. European Association for Cardiothoracic Surgery-Society of Thoracic Surgeons Joint Congenital Heart Surgery Nomenclature and Database Committee. Computerized outcomes analysis for congenital heart disease. Curr Opin Pediatr 2005; 17: 586-591; Review.

31. [http://www.ihtsdo.org/about-us/history/], accessed July 2007.

32. Gibbs JL, Monro JL, Cunningham D, Rickards A. Survival after surgery or therapeutic catheterisation for congenital heart disease in children in the United Kingdom: analysis of the central cardiac audit database for 2000-1. Brit Med J 2004; 328: 611-615. 